\title{
QT interval variables from 24 hour electrocardiography and the two year risk of sudden death
}

\author{
A Algra, J G P Tijssen, J R T C Roelandt, J Pool, J Lubsen
}

\begin{abstract}
Objective-To study the effects of variability in the duration of the QT interval corrected for heart rate (QTc) on the occurrence of sudden death.

Design-Nested case-referent study.

Subjects-Cohort of 6693 consecutive patients who underwent 24 hour electrocardiography and were followed up for two years. Risk implications of QTc interval variables were studied in patients without evidence of cardiac dysfunction or of an intraventricular conduction defect (104 died suddenly and 201 patients were randomly drawn from the study cohort).
\end{abstract}

Main outcome measures-Mean QTc interval duration and variation in QTc duration over time correlated with occurrence of sudden death.

Results-Patients with a prolonged mean QTc over 24 hours ( $\geqslant 440 \mathrm{~ms}$ ) had a $2 \cdot 3$ times $(95 \%$ confidence interval $1 \cdot 3$ to $4 \cdot 5)$ higher risk of dying suddenly than patients with a normal mean QTc (400-440 ms); patients with a shortened mean QTc ( $<400 \mathrm{~ms}$ ) also had a higher risk (relative risk $2.4(1.4$ to $4 \cdot 3)$ ). Patients with low $(<20 \mathrm{~ms})$ and high ( $\geqslant 25 \mathrm{~ms}$ ) long term variation in QTc duration had an increased risk of dying suddenly compared with those with intermediate variation (20-25 ms) (relative risks $2.2(1.2$ to 4.2$)$ and $2.3(1.4$ to $4 \cdot 2)$ respectively). The relative risks for low and high short term variation were not significantly raised.

Conclusions-A prolonged and a shortened mean QTc interval over 24 hours was associated with a more than twofold risk of sudden death compared with intermediate mean QTc values (400-440 ms). Neither short nor long term variability in QTc had a distinct relation with the risk of sudden death.

(Br Heart f 1993;70:43-48)

Although ample evidence suggests that disturbance of autonomic nervous system activity is accompanied by an increased risk of sudden death, ${ }^{1}$ no large epidemiological studies have been undertaken on this subject. Extensive studies of autonomic nervous system function in high risk patients were recommended by the fourth task force on non-pharmacological interventions in sudden cardiac death which was set up at the American Heart Association's conference on behavioural medicine and cardiovascular disease in $1985 .^{2}$

Part of the effects of the autonomic nervous system on the occurrence of sudden death is related to the balance between the activity of the left and right sympathetic nervous system and another part to the balance of the sympathetic and parasympathetic nervous systems. In patients with the long QT syndrome clinical and experimental data support the theory that an imbalance of the left and right cardiac sympathetic innervation forms the pathophysiological basis for the frequently occurring ventricular arrhythmias. Primary hypoactivity of the right cardiac sympathetic nerves results in reflex hyperactivity of the left nerves. ${ }^{3}$ Also, a prolonged QT interval corrected for heart rate (QTc) measured during standard 12 lead electrocardiography is associated with a twofold risk of sudden death. ${ }^{45}$

We surmised that if sudden death is precipitated by a disturbance of the balance between left and right sympathetic activity in damaged hearts these disturbances as evidenced by variation in QTc duration might identify patients with an increased risk of dying suddenly. We therefore studied the risk of sudden death associated with variability in the QTc interval in a cohort of 6693 consecutive patients who underwent 24 hour ambulatory electrocardiography.

\section{Patients and methods}

STUDY COHORT

All 6693 consecutive patients who had 24 hour electrocardiography in one of the four participating Rotterdam hospitals between 1 August 1980 and 31 December 1984 were included in this study. Indications for 24 hour electrocardiography were evaluating symptoms potentially related to cardiac arrhythmias (palpitation, dizziness, syncope, angina) $(65 \%)$, the effect of antiarrhythmic treatment $(8 \%)$, or risk after myocardial infarction $(10 \%)$ or searching for a cardiac cause of transient ischaemic attacks or strokes (7\%). Three quarters of the 24 hour electrocardiograms were recorded in outpatients.

\section{FOLLOW UP}

Deaths were recorded up to two years after 24 hour ambulatory electrocardiography. Follow up was complete in $99.5 \%$ of the 
patients: 716 patients died $(10.7 \%)$. The cause and circumstances of death were determined from the records of general practitioners and hospitals. Patients were considered to have died suddenly if death had been observed and had occurred within one hour after new or increasing symptoms and provided that its cause was cardiovascular. Also, patients who unexpectedly died during sleep or who died while unobserved with circumstantial evidence suggesting a sudden death were considered to have died suddenly. All records of cases of sudden death were independently verified by two senior cardiologists.

\section{CLINICAL BASELINE DATA}

Baseline characteristics were retrospectively collected for the 245 patients who died suddenly and for a random sample of 268 patients from the complete study cohort. Information on the following characteristics at the time of 24 hour electrocardiography was collected from the patients' records: known cardiovascular risk indicators, cardiovascular history, results of cardiovascular function tests (if available) and of routine laboratory studies, and current drug use. Cardiac dysfunction was considered to be present when there was a history of symptoms and signs of pump failure or the ejection fraction was less than $40 \%$ at cineangiographic or radionuclide ventriculography.

\section{RECORDING AND ANALYSIS OF 24 HOUR}

ELECTROCARDIOGRAMS

24 Hour electrocardiograms were recorded with the use of Oxford Medical Instruments recorders of the AM and FM recording type. Two bipolar leads were used; a V2 equivalent was derived from two electrodes located at the sternum and a V5 equivalent from an electrode at the sternum and one at the V5 position.

After detection of QRS complexes during a first pass analysis ${ }^{6}$ QT intervals were measured during a second pass of automated computer aided analysis ${ }^{7}$ and were reviewed and corrected manually. The lead in which the duration of the QT interval had the strongest correlation with the duration of the RR interval was selected for QT editing because the quality of the QT measurements in this lead was considered to be best. As RR and QT interval variabilities were the main determinants of study, these variables were used as the criteria to select three half hour episodes to limit editing time. From the 48 half hour episodes the episode with maximum $R R$ variability (standard deviation of the averages per minute of the RR intervals in a half hour episode), the episode with maximum QT interval variability (the standard deviation of all QT intervals in a half hour episode), and the episode with the shortest TQ intervals (average of all TQ intervals in a half hour episode) were selected. The episode with the shortest TQ interval was selected because of the proximity of the end of repolarisation and the start of the next depolarisation, a high risk state in the initiation of ventricular
Table 1 Definitions of $Q T c$ duration and variability in 24 hour electrocardiograms

\begin{tabular}{|c|c|}
\hline QTc variable & Definition \\
\hline Duration: & $\begin{array}{l}\text { Mean of all QTc means per minute over } \\
24 \text { hours }\end{array}$ \\
\hline Maximum & $\begin{array}{l}\text { Maximum QTc mean per minute over } \\
24 \text { hours }\end{array}$ \\
\hline Minimum & $\begin{array}{l}\text { Minimum QTc mean per minute over } \\
24 \text { hours }\end{array}$ \\
\hline$>440 \mathrm{~ms}$ & $\begin{array}{l}\text { Mean of percentages per minute with QTc } \\
>440 \text { ms over } 24 \text { hours }\end{array}$ \\
\hline \multicolumn{2}{|l|}{ Variability: } \\
\hline Short term & $\begin{array}{l}\text { Mean of all SDS of QTc means per } \\
\text { minute over } 24 \text { hours }\end{array}$ \\
\hline Long term & $\begin{array}{l}\text { SD of QTc means per minute over } \\
24 \text { hours }\end{array}$ \\
\hline
\end{tabular}

arrhythmias. QT intervals not selected for review in one of the three half hour episodes were adjusted for the mean difference of QT measurements before and after review in the three selected half hour episodes in the particular 24 hour electrocardiogram

All QT analyses were performed using heart rate corrected QT intervals (QTc). ${ }^{8}$ Table 1 shows the definitions of the parameters of QTc interval duration and variation. QTc was calculated only if the current and previous $Q R S$ complex were of supraventricular origin, while a running $R R$ average with $1 / 8$ update was used in the computation. (With every new RR interval the new running $R R$ average is calculated as the sum of $7 / 8$ th of the previous average and 1/8th of the current $R R$ interval.)

For comparison with their equivalent lead on the 24 hour electrocardiogram QTc intervals were determined in leads V2 and V5 of standard 12 lead electrocardiograms recorded at the time of 24 hour electrocardiography in all patients who died suddenly and in the patients from the random sample.

\section{DATA ANALYSIS}

In the data analysis four patients with unanalysable 24 hour electrocardiograms were excluded (all had died suddenly). From the QTc analyses patients with an intraventricular conduction defect (Minnesota code $7^{9}$ ) at the standard 12 lead electrocardiogram recorded at the time of 24 hour electrocardiography were excluded. Also patients with evidence of cardiac dysfunction were excluded because analyses of the standard 12 lead electrocardiogram QTc showed no relation between prolonged QTc and sudden death in these patients, ${ }^{5}$ leaving 104 cases of sudden death and 201 patients from the random sample.

The effect of a QT variable on the risk of dying suddenly was expressed as the relative risk, which is equal to the risk of sudden death among patients with the characteristic divided by that among patients without the characteristic. The risk of sudden death among patients with the characteristic was obtained as the number of sudden deaths with the characteristic divided by the (estimated) total number of patients with the characteristic. The total number of patients 
Table 2 Patient characteristics at time of 24 hour electrocardiography in patients who died suddenly and in those from the random sample of the study cohort. Values are percentages

\begin{tabular}{lll} 
& $\begin{array}{l}\text { Sudden } \\
\text { deaths } \\
(n=104)\end{array}$ & $\begin{array}{l}\text { Referents } \\
(n=201)\end{array}$ \\
& 63 & 81 \\
Outpatient & 73 & 58 \\
Men & 78 & 49 \\
Age $>60$ years & 50 & 29 \\
History: & 53 & 26 \\
Angina & 50 & 36 \\
Myocardial infarction & 45 & 30 \\
Supraventricular arrhythmias & 6 \\
Ventricular arrhythmias & 12 & 51 \\
Atrioventricular conduction defects & 25 & 45 \\
Palpitations & 46 & 17 \\
Dizziness & 30 & 5 \\
Syncope & 3 & 15 \\
Coronary bypass surgery & 8 \\
Transient ischaemic attack or stroke & 34 & 8 \\
Intermittent claudication & 14 & 8 \\
Diabetes mellitus & 16 & 38 \\
Cigarette smoking & 33 & \\
Drug use: & 29 & 12 \\
Digitalis & 42 & 28 \\
$\beta$ blockers & 39 & 17 \\
Diuretics & 9 & 10 \\
Antiarrhythmic drugs (class): & 9 \\
$\quad 1$ & 4 & 3 \\
3 & 7 & 7 \\
\hline 4 & & \\
\hline
\end{tabular}

with the characteristic was obtained by extrapolating the findings for the QT variable in the sample to the whole study cohort - that is, by multiplying the number of patients with the characteristic in the sample by $6693 / 268$, the inverse of the sampling fraction. In this calculation a correction factor of $241 / 245$ was used to compensate for the exclusion of four cases. The risk of sudden death among patients without the characteristic was obtained similarly. The $95 \%$ confidence

Table 3 Occurrence of sudden death in relation to several variables of QTc duration in 24 hour electrocardiograms in patients without intraventricular conduction defects or evidence of cardiac dysfunction

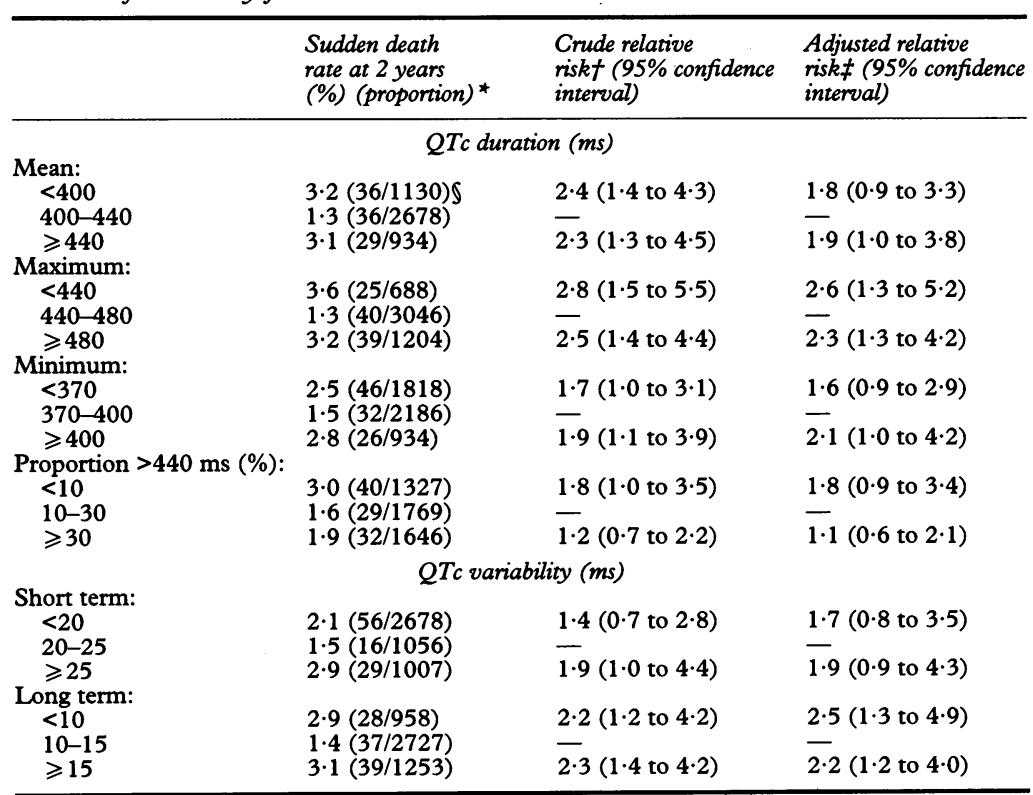

*Denominator estimated as number of patients from random sample multiplied by inverse of sampling fraction, 6693/268, and corrected by $241 / 245$ because four cases of sudden death were excluded.

†Ratio of sudden death rates in which the rate of a category indicated by a missing relative risk was taken as reference.

$\ddagger$ Adjusted for age and history of myocardial infarction and calculated by logistic regression.

§Numerators and denominators do not always add up to 104 and 4938 respectively because episodes of poor signal quality in $\mathbf{2 4}$ hour electrocardiograms were excluded. interval of the odds ratio was used and calculated from the data laid out for a case-control study. ${ }^{10}$

We used stratified analysis and logistic regression to calculate adjusted relative risks to assess the influences of independent risk factors of sudden death that were unequally distributed between patients with and without a given QT variable.

\section{Results}

Table 2 shows the distribution of baseline characteristics of the 104 patients who died suddenly and the 201 patients from the random sample selected for the QTc data analysis. Risk factors for sudden death were more prevalent among those who died suddenly-for example, age and history of myocardial infarction, angina, and diabetes mellitus. Also, patients from the random sample took fewer amounts of digitalis, $\beta$ blockers, and diuretics, but the use of antiarrhythmic drugs was similar in both groups of patients.

Table 3 shows the occurrence of sudden death in patients for several QTc variables. Crude analysis (unadjusted relative risks) shows that patients with a shortened and prolonged mean QTc had a more than twofold risk of dying suddenly compared with patients with mean QTc values between 400 and $440 \mathrm{~ms}$. The same pattern was found for the other duration variables: maximum and minimum QTc and the percentage QTc greater than $440 \mathrm{~ms}$. The increased risks associated with a shortened and a prolonged QTc were found to be independent of heart rate. When the analysis was restricted to the three half hour episodes with manually edited QT measurements the same picture emerged. The risk increase related to the QTc variables was more pronounced in the V2 equivalent lead than in the V5 equivalent lead. For short term and long term QTc variability an

Table 4 Occurrence of sudden death in relation to $Q T c$ duration in leads V2 and V5 of 12 lead resting electrocardiograms recorded at time of 24 hour electrocardiography in patients without intraventricula conduction defects or evidence of cardiac dysfunction

\begin{tabular}{ll}
\hline & Sudden death \\
rate at 2 years Relative risk $\dagger$ \\
$Q T c$ duration (ms) & (\%) (proportion) * \\
(95\% confidence interval)
\end{tabular}

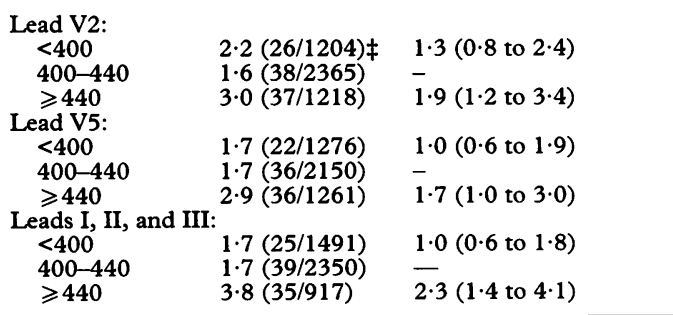

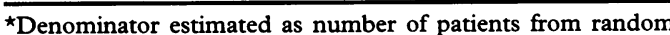
* Denominator estimated as number of patients from random and corrected by $241 / 245$ because four cases of sudden death were excluded.

were excluded.
tRatio of sudden death rates in which the rate of a category TRatio of sudden death rates in which the rate of a cate
indicated by a missing relative risk was taken as reference. $\ddagger$ Numerators and denominators do not always add up to 104 and 4938 respectively because arrhythmias in standard 12 lead electrocardiograms sometimes precluded measurement of QTc. 
increased risk was found in both high and low extremes, as well as when the analysis was restricted to the three edited episodes.

During the assessment of the influence of other risk factors for sudden death on the crude relative risk estimates we found that the combination of age and history of myocardial infarction altered the estimates slightly (table 3 , right column); addition of other patient characteristics, including drug use and serum potassium concentration, to the logistic models yielded no further relevant changes.

The data in Table 4 on QTc duration in 12 lead resting electrocardiograms recorded at the time of 24 hour electrocardiography show no increased risk of sudden death in patients with a shortened QTc in the combined leads I, II, and III and in single lead V5; a trend was seen in single lead V2 (relative risk 1.3).

\section{Discussion}

\section{QTC INTERVAL DURATION}

Prolonged mean QTc duration over 24 hours of greater than $440 \mathrm{~ms}$ was related to a more than twofold risk of sudden death compared with QTc durations between 400 and 440 ms. Prolonged minimum and maximum QTc duration (for definition see table 1) showed comparable increases in risk. These findings agree with previous results from standard 12 lead electrocardiography in the same patients. ${ }^{5}$ However, a striking new finding was that patients with a mean QTc duration of less than $400 \mathrm{~ms}$ also had a more than twofold risk of dying suddenly compared with patients with intermediate QTc durations (400-440 ms). This finding was unexpected because earlier studies on the risk implications of QTc duration, including ours, ${ }^{5}$ did not indicate that shortened QTc was related to an increased risk of sudden death. ${ }^{5}$

The finding contradicts the idea that prolonged QTc is an electrocardiographic equivalent of heterogenous prolongation of repolarisation, ${ }^{11}$ which itself predisposes to the occurrence of malignant ventricular arrhythmias and sudden death. Shortened QTc would indicate (more) homogeneous repolarisation and hence a decreased risk of sudden death. Explanations for the discrepancy between the findings from 24 hour and standard 12 lead electrocardiography must be sought in the differences between these methods.

Firstly, during standard 12 lead electrocardiography the patient is in a static state compared with that during dynamic 24 hour electrocardiography; this has implications for the use of the Bazett formula. ${ }^{8}$ This difference, however, is unlikely to explain our observation on shortened QTc because we found that QTc interval duration at resting hours during night time (0001-0500) had the same risk effects as during the complete 24 hours. The validity of the Bazett formula under static circumstances should be critically reassessed. Many alternative formulas for the heart rate correction of the QT interval have been proposed, ${ }^{12}$ but QTc according to Bazett is the most widely used, for example, to diagnose the long QT syndrome. ${ }^{3}$ The U shaped risk curve of QTc interval duration also emerged in the subgroup with lowest mean heart rate. At these heart rates only minor differences exist between QT and QTC interval duration. We therefore concluded that our observation on shortened QTc is unlikely to be explained by the use of the Bazett formula.

Secondly, all studies on QTc duration derived from standard 12 lead electrocardiography, including ours, used standard leads I, II, and III. During 24 hour electrocardiography, however, bipolar leads were used which correspond best with the unipolar leads V2 and V5. Thus, it is more appropriate to compare QTc measurements in leads V2 and V5 with their equivalents from a 24 hour electrocardiogram. QTc in lead V5 did not show an increased risk for patients with shortened QTc while in lead V2 a trend (relative risk of 1.3) was observed. Theoretically a long QT interval in one lead may concur with a short QT interval in another lead if the last part of repolarisation in that lead is isoelectric. Our data showed that the relation of shortened QTc to an increased risk of sudden death was most pronounced in the anteriorly placed leads: V2 in standard 12 lead electrocardiography and in the V2 equivalent lead in 24 hour electrocardiography. Thus, in some patients with prolonged repolarisation the terminal phase may occur in the frontal plane only, perpendicular to the lead axes of anteriorly located leads and hence invisible from there.

Thirdly, the frequency characteristics of 24 hour electrocardiographic systems are limited. ${ }^{13}$ This might lead to distorted registration of repolarisation and a systematic error in measuring QT duration. This does not, however, explain the $U$ shaped risk curve.

Fourthly, mean QTc over 24 hours is based on QTc computations in which QT intervals measured by computer and a running $R R$ average are used, a large range of heart rate is covered, and an average over 24 hours is determined compared with the simple calculation of QTc from standard resting 12 lead electrocardiography, in which one QT and one RR interval are used. Nevertheless, those computational differences probably do not play a major part because shortened QTc in the three edited episodes had the same risk implications. Moreover, the same trend was found for the other QTC duration variables, whereas the effect did not depend on heart rate.

We conclude therefore that we may have observed a true pathophysiological phenomenon. If re-entry is responsible for sudden death and re-entry is more likely to occur with heterogeneous repolarisation and a short excitation wavelength (the product of refractory period and conduction velocity) then at least two explanations may be given for an increased risk of sudden death with a shortened QT interval. Firstly, heterogeneous

:
.

(n)

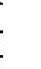

.


repolarisations may seem to be complete from anteriorly located electrodes. This is an artefact because terminal repolarisation occurs in a plane which cannot be observed from this electrode. A discrepancy between QT duration from different leads may then be interpreted as evidence of heterogeneous repolarisation, one of the conditions for reentry arrhythmias. Secondly, a short QT interval may be the result of a short refractory period, which would yield a short excitation wavelength predisposing to re-entry. ${ }^{14}$

QTC INTERVAL VARIABILITY

The percentage of QTc intervals greater than $440 \mathrm{~ms}$ - that is, the percentage of prolonged intervals - was taken as a measure of the number of episodes during which the patient would be in a state of high risk of dying suddenly. Based on observations on QTc duration in standard 12 lead electrocardiograms and data presented by the group of Bayès de Luna $^{15}$, we expected that a high percentage of QTc intervals greater then $440 \mathrm{~ms}$ would be related to a high sudden death rate. This is not supported by our data. This finding may be attributed to the limited relation of prolonged mean QTc duration over 24 hours to sudden death. The variables describing QTc variation can be interpreted as a measure of the number and duration of episodes in which extreme QTc durations occur, hence the number and duration of high risk episodes. No clear relation of high variation with sudden death, however, emerges from our data, probably for the same reason as mentioned before: the lack of a strong relation of prolonged QTc with sudden death. Our observations disagree with those of Schwartz et al, who noted higher variation of QTc duration in bimonthly repeated standard 12 lead electrocardiograms in patients who died suddenly during a seven year follow up after myocardial infarction than in patients who survived the follow up. ${ }^{16}$ In this study, however, prolonged QTc was related to sudden death.

\section{STUDY DESIGN}

We chose a nested case-referent design for efficiency considerations. ${ }^{1718}$ Although baseline characteristics were collected only for a small subset of patients, the essential findings were produced with the same level of precision as in a conventional follow up study. ${ }^{17} 18$ We calculated a $95 \%$ confidence interval (based on the observed data) that ranged from 1.4 to 4.3 for the relative risk of a mean QTc interval of less than $400 \mathrm{~ms}$ for sudden death. If we had measured mean QTc in all patients fulfilling the selection criteria for this study - that is, if we had observed the upper two lines of table 3-the confidence interval would have ranged from 1.5 to 3.9 .

\section{IMPLICATIONS}

Given the elaborate procedure used in this study to obtain QTc variables from 24 hour electrocardiography and the limited risk implications of these variables with regard to the occurrence of sudden death, measurement of QTc in 24 hour electrocardiograms may have limited value in routine clinical practice. The finding, however, that shortened mean QTc derived from 24 hour electrocardiograms is related to an increased risk of sudden death may stimulate new research on the effect of electrode localisation on QTc duration and its pathophysiological mechanisms.

\section{LIMITATIONS OF THE STUDY}

The criteria to classify the cases of sudden death were pragmatic because information on cause and circumstances of death had to be obtained between one and four years after death. More strict criteria may be used only when cases are ascertained concurrently. All available data were studied in detail, and three investigators (AA, JP, and JRTCR) had to agree on the classification.

The occurrence of sudden death in the cohort was conditional on interventions after 24 hour electrocardiography. Therefore the findings during routine analysis of the 24 hour electrocardiograms may have influenced intervention strategies. This would jeopardise the validity of estimating the effect of the electrocardiographic variables on sudden death. In clinical practice, however, only extremely prolonged QTc would lead to specific intervention; the other electrocardiographic variables (heart rate variability and QT interval variability) are not generally used in clinical practice. Thus, our estimates of relative risk are unlikely to have been influenced by the findings on 24 hour electrocardiography. Nevertheless, when interpreting the results of our study it should be remembered that the findings are conditional on the treatment given, which may have been influenced by the electrocardiographic findings.

\section{CONCLUSIONS}

Both prolonged and shortened mean QTC derived from 24 hour electrocardiography are related to a twofold risk of sudden death compared into intermediate QTc values (400-440 ms). QTc variability has no clear relation to sudden death.

We thank Dr M J Janse, University of Amsterdam, and Dr P Coumel, Hôpital Lariboisière, Paris, for their critical com ments; Mr J L H Le Brun and Mrs S van der Does-van der ments; Mr J L H Le Brun and Mrs S van der Does-van der Linden for their great help with the retrieval and coding of the
patient records; Mr J L H Le Brun also for the many hours he spent on the analysis of the 24 hour electrocardiograms; and the cardiology staff of the University Hospital RotterdamDijkzigt, the Bergwegziekenhuis, the Sint Franciscus Gasthuis, and the Zuiderzickenhuis, Rotterdam, for their cooperation. This work was supported by grants 83.075 and 37.002 from the Netherlands Heart Foundation, The Hague, and a grant from Cardiolab, Rotterdam.

1 Zipes DP, Levy MN, Cobb LA, et al. Task Force 2: Sudden cardiac death. Neural-cardiac interactions. Circulation 1987;76(suppl I):I-202-7.

2 Schwartz PJ, Randall WC, Anderson EA, et al. Task Force 4: Sudden cardiac death. Nonpharmacologic interventions. Circulation 1987;76(suppl I):I-215-9.

3 Schwartz PJ. Idiopathic long QT syndrome: progress and questions. Am Heart f 1985;109:399-411.

4 Locati E, Schwartz PJ. Prognostic value of QT interval prolongation in post myocardial infarction patients. Eur Heart $f 1987 ; 8$ (suppl A):121-6. 
5 Algra A, Tijssen JGP, Roelandt JRTC, Pool J, Lubsen J. QTc prolongation measured by standard 12 lead electrocardiography is an independent risk factor for sudden death due

6 Ripley KL, Okkerse RJ, Engelse WAH, Vinke RVII, Zeelenberg C. Implementation of ARGUS $/ 2 \mathrm{H}$ at the Thorax centrum. Proceedings Computers in Cardiology 1980;7:135-8.

7 Algra A, Le Brun H, Zeelenberg C. An algorithm for computer measurement of QT intervals in the 24 hou ECG. Proceedings Computers in Cardiology 1986;13: $117-9$

8 Bazett HC. An analysis of the time-relations of electrocardiograms. Heart fournal 1920;7:353-70.

9 Prineas RJ, Crow RS, Blackburn H. The Minnesota code Manual of electrocardiographic findings. Standards and procedures for measurement and classification. Boston: John cedures for measuremen

10 Woolf $B$. On estimating the relation between blood group and disease. Ann Hum Genet 1955;19:251-3.

11 Corr PB, Yamada KA, Witkowski FX. Mechanisms controlling cardiac autonomic function and their relation to arrhythmogenesis. In: Fozzard HA et al eds. The heart and cardiovascular system. Scientific foundations.
New York: Raven, 1986;1343-403

12 Puddu PE, Jouve R, Mariotti S, et al. Evaluation of 10 QT prediction formulas in 881 middle-aged men from the
seven countries study: emphasis on the cubic root Fridericia's equation. $¥$ Electrocardiol 1988;21:219-29.

13 Bragg-Remschel DA, Anderson CM, Winkle RA. Frequency response characteristics of ambulatory ECG monitoring systems and their implications for ST segment analysis. Am Heart F 1982;103:20-31.

14 Rensma PL, Allessie MA, Lammers WJEP, Bonke FIM, Schalij MJ. Length of excitation wave and susceptibility to reentrant atrial arrhythmias in normal conscious dogs. Circ Res 1988;62:395-410.

15 Laguna $P$, Caminal $P$, Marti V, et al Automatic measurement of corrected QT (QTc) as a marker of malignant ventricular arrhythmias in postmyocardial infarction patients [abstract]. Eur Heart $\mathcal{f} 1989 ; 10$ (suppl): 98

16 Schwart PJ, Wolf S. QT interval prolongation as predictor of sudden death in patients with myocardial infarction. Circulation 1978;57:1074-7.

17 Rothman KJ. Modern epidemiology. Boston: Little, Brown, 1986:61-86.

18 Kleinbaum DG, Kupper LL, Morgenstern H. Epidemiologic research. Belmont: Lifetime Learning Publications, 1982:71. 